Communications in Physics, Vol. 25, No. 3 (2015), pp. 283-290

DOI: $10.15625 / 0868-3166 / 25 / 3 / 5561$

\title{
RESEARCH OF SOIL MOISTURE RETRIEVAL ALGORITHMS FOR PROCESSING RADIOMETRY DATA
}

\author{
VO THI LAN ANH, NGO TUAN NGOC, AND DOAN MINH CHUNG \\ Space Technology Institute, Vietnam Academy of Science and Technology \\ K. G. KOSTOV \\ Institute of Electronics, BAS, Bulgaria \\ E-mail:vtlanh@sti.vast.vn \\ Received 26 November 2014 \\ Accepted for publication 27 September 2015
}

\begin{abstract}
Since 2012, the experts of Space Technology Institute have carried out the field experiments to obtain a high-resolution dataset of microwave radiometers for land surface parameters (soil moisture, soil temperature, vegetation water content), in order to improve the soil moisture retrieval methodology. L-band radiometers were used for measuring the brightness temperature of the bare soil. Field experiments for passive microwave remote sensing of soil moisture were carried out in Hoai Duc District in 2012. L-band microwave radiometers were used for measuring the microwave emission of bare agricultural fields. The radiometers, which are used for soil moisture measurement, worked well during the experimental campaign and produced volumetric soil moisture estimates that compared well with the ground-truth measurements. Explanations for the observed discrepancies are presented. The experimental results showed that the model of Choudhury et al. for surface roughness correction provides a better fit to radiometric data over the angular range between $20^{\circ}$ and $50^{\circ}$ for $n=0$ (i.e., the $\cos ^{2} \theta$ factor in the exponential in (15) is suppressed). Based on the results of the experiments conducted over two experimental sites with different soils, namely sandy loam at Hoai Duc Agrometeorologyl Center, it may be concluded that the testing of both the radiometric equipment and the method for soil moisture retrieval was very successful, and the main goal of the experiments was fulfilled.
\end{abstract}

Keywords: soil moisture retrieval, dielectric model, passive microwave remote sensing.

\section{INTRODUCTION}

Soil moisture is a key factor playing a vital role in the functioning of ecosystems and in mass and energy exchange processes occurring at the soil-atmosphere interface. Soil moisture information is important in a variety of disciplines (e.g., hydrology, agriculture, and meteorology). Attempts to monitor world food supply, predict watershed run-off, and model boundary layer heat and moisture conditions are a few of many specific areas in which surface and subsurface soil moisture information is needed. Soil moisture is difficult to measure large areas due to its high spatial and temporal variability. Microwave remote sensing systems are widely used for remote sensing of the earth surface due to the nearly all-weather, day/night capability and relatively large penetration depth. The basis for microwave remote sensing of soil moisture is mostly dependent 
the soil's dielectric properties and its moisture content due to the large contrast between the dielectric constant of water and that of dry soil. Major factors determining the microwave radiation from a soil surface are: soil moisture, soil texture and surface roughness, soil temperature, and vegetation $[1,5]$. Microwave remote sensing techniques have been applied for estimating the surface soil moisture in a variety of environmental conditions. We believe that a great deal of the published experimental data relating to the L-band emissivity for the average volumetric soil moisture in the top $5 \mathrm{~cm}$ of soil is due to the influence of the near-surface moisture (NSM) profile [10]. Now it is accepted that at L-band depth of the soil layer is from 2 to $5 \mathrm{~cm}[3,10,13]$, depending on the near-surface moisture (NSM) profile [10] and surface roughness state.

This paper briefly describes the passive microwave remote sensing on soil moisture and effects of surface roughness to the land surface. The paper also presents soil moisture retrieval algorithms and modeling approaches in Vietnam Space Technology Institute.

\section{SOIL MOISTURE RETRIEVAL FROM PASSIVE MICROWAVE REMOTE SENSING}

\section{II.1. Emission Model}

The microwave brightness temperature of a soil medium is governed by the dielectric and temperature depth profiles. Due to the strong dependence of the soil dielectric constant on its moisture content, the brightness temperature $T_{B}$ exhibits a strong sensitivity to soil moisture content.

The p-polarized brightness temperature of a soil medium with smooth surface observed at angle $\theta$ may be expressed as

$$
T_{B p}(\theta)=\int_{-\infty}^{0} T(z) F_{p}\{\varepsilon(z), \theta\} d z,
$$

where $\varepsilon(z)$ is the dielectric constant depth profile and $T(z)$ is the soil temperature depth profile. The function $F P\{\varepsilon(z), \theta\}$ is in a form of the weighting function, and indicates the relative contributions of the subsurface temperatures to the overall brightness temperature. Exact analytical solutions to equation (1) exist only for a few simple forms of the soil dielectric constant and temperature profiles. Equation (1) is a form commonly used in remote sensing and its inversion to obtain soil profile parameters is a problem. One reason for this problem is that the temperature and soil moisture profiles are not independent, and the inversion problem is nonlinear. Njoku and Kong [4] used a stratified model to compute the microwave brightness temperature of a soil medium with known soil moisture and temperature profiles. The stratified model [4] is not suitable for remote sensing applications since it requires detailed knowledge of the soil moisture and temperature profiles. Therefore, a simpler model for calculating the brightness temperature of soil is needed for remote sensing applications.

Schmugge and Choudhury [16] proposed a simpler radiative transfer model for calculating the brightness temperature of soil. We shall refer to it as a zero-order model [5]. The brightness temperature is given by

$$
T_{B p}(\theta)=\left[1-R_{p}(\theta)\right] T_{e f f}(\theta),
$$


where $R_{p}(\theta)$ is the Fresnel reflectivity for the air-soil surface and $T_{e f f}(t \theta)$ is an effective radiating temperature given by

$$
T_{e f f}(\theta)=\sec \theta_{1} \int_{0^{+}}^{\infty} T(z) k_{a}(z) \exp \left(-\int_{0}^{z} k_{a}\left(z^{\prime}\right) \sec \theta_{1} d z^{\prime}\right) d z .
$$

The angle $\theta_{1}$ is the refraction angle in the soil medium and is related to $\theta$ by Snell's law [5], and $z=0+$ corresponds to a point located just below the soil surface, and $k_{a}(z)$ is the soil absorption profile. For an isothermal soil medium $\left\{T(z)=T_{S}\right\}$, equation (3) reduces to $T_{\text {eff }}(\theta)=$ $T_{S}$ for any nonzero profile $k_{a}(z)$; consequently, equation (2) reduces to

$$
T_{B p}(\theta)=e_{p}(\theta) T_{S}=\left[1-R_{p}(\theta)\right] T_{S},
$$

for the simplest soil-emission configuration represented by a homogeneous isothermal soil medium with a plane air-soil boundary. $T_{S}$ is the soil thermometric temperature, and $e_{p}(\theta)$ is the $p$-polarized specular emissivity of the soil surface. The reflectivity $R_{p}(\theta)$ can be calculated by the Fresnel equations:

$$
\begin{gathered}
R_{h}(\theta)=\left(\frac{\cos \theta-\sqrt{\varepsilon-\sin ^{2} \theta}}{\cos \theta+\sqrt{\varepsilon-\sin ^{2} \theta}}\right)^{2}, \\
R_{v}(\theta)=\left(\frac{\varepsilon \cos \theta-\sqrt{\varepsilon-\sin ^{2} \theta}}{\varepsilon \cos \theta+\sqrt{\varepsilon-\sin ^{2} \theta}}\right)^{2} .
\end{gathered}
$$

\section{II.2. Dielectric Model}

At a given frequency, the dielectric constant $\varepsilon$ of soil is, in general, a function of several quantities including moisture content, bulk density, soil textural composition, soil temperature, and salinity. Among these quantities, moisture content has a greatest influence on $\varepsilon$. A wet soil medium is a mixture of soil particles, air voids, and liquid water. The water contained in the soil usually is divided into two fractions - bound water and free water. Electromagnetically, a soil medium is, in general, a four-component dielectric mixture consisting of bulk soil, air, bound water, and free water. Due to the high intensity of the forces acting upon it, a bound water molecule which interacts with an incident electromagnetic wave is dissimilar to that of a free water molecule, thereby exhibiting a dielectric dispersion spectrum that is very different from that of free water. The complex dielectric constants of bound and free water are the functions of frequency, physical temperature and salinity [5]. Hence, the dielectric constant of the soil mixture is, in general, a function of: 1) the frequency, temperature and salinity; 2) the total volumetric water content; 3 ) the relative fractions of bound and free water, which are related to the soil surface area per unit volume; 4) the bulk density of soil; 5) the shape of the soil particles; 6) the shape of the water inclusions. Different mixing models are proposed for calculating the dielectric constant $\varepsilon$ of soil [6-8]. The model of Wang and Schmugge [6] will be used in this paper. The soil complex dielectric constant $\varepsilon$ is computed by using a mixing equation that considers the contributions of the soil particles, air, and water as follows [6]:

$$
\varepsilon=m_{v} \varepsilon_{x}+\left(P-m_{v}\right) \varepsilon_{a}+(1-P) \varepsilon_{r}, \quad m_{v} \leq W_{t}
$$


with

$$
\varepsilon_{x}=\varepsilon_{i}+\left(\varepsilon_{w}-\varepsilon_{i}\right)\left(m_{v} / W_{t}\right) \gamma
$$

and

$$
\varepsilon=W_{t} \varepsilon_{x}+\left(m_{v}-W_{t}\right) \varepsilon_{w}+\left(P-m_{v}\right) \varepsilon_{a}+(1-P) \varepsilon_{r}, \quad m_{v}>W_{t}
$$

with

$$
\varepsilon_{x}=\varepsilon_{i}+\left(\varepsilon_{w}-\varepsilon_{i}\right) \gamma
$$

Here $m_{v}$ is the volumetric soil moisture, $P$ is the porosity of the dry soil, $\varepsilon_{a}, \varepsilon_{w}, \varepsilon_{r}$, and $\varepsilon_{i}$, in sequential order, are the dielectric constants of air, water, rock, and ice. $\varepsilon_{x}$ stands for the dielectric constant of the initially absorbed water. $W_{t}$ is transition moisture and $\gamma$ is a parameter.

The porosity $P$ of a dry soil is defined as

$$
P=1-\left(\rho_{s} / \rho_{r}\right),
$$

where $\rho_{s}$ is the density of the dry soil and $\rho_{r}$ is the density of the associated solid rock.

$W_{t}$ and $\gamma$ are given as follows:

$$
\gamma=-0.57 W P+0.481
$$

and

$$
W_{t}=0.49 W P+0.165 \text {. }
$$

The wilting point $W P$ (in terms of volumetric moisture content) is given as

$$
W P=0.06774-0.00064 S F+0.00478 C F,
$$

where $S F$ and $C F$ are the sand and clay contents in percent of dry weight of a soil.

\section{II.3. Surface Roughness Correction}

When the soil surface is rough, the expressions for soil reflectivity must be modified. Theoretical expressions for reflectivity from rough surface have been developed by using statistical parameters of the surface, such as the standard deviation of surface height $\sigma$ and surface correlation length $l$. Many theoretical and experimental investigations were conducted during the past two decades [2-13] and several simpler models were proposed for correcting the roughness effects on microwave emission from soils [2], and [9].

Choudhury et al. [2] proposed a model using a single surface-roughness parameter, the standard deviation of surface height $\sigma$. According to this model, the rough surface reflectivity $R_{r}$ is given by

$$
R_{r p}(\theta)=R_{p}(\theta) \exp \left(-h \cos ^{2} \theta\right)
$$

where $R_{p}(\theta)$ is the Fresnel reflectivity and

$$
h=4 k^{2} \sigma^{2}
$$

is called a roughness parameter.

The model of Choudhury et al. [2] was applied to radiometric measurements made over bare-soil fields with various roughness scales [2, 5, 7, 13],. By treating $h$ as a free parameter for a given surface, fairly good agreement was obtained between the measured values of $T_{B}$ and those used calculations (13). The selected $h$ values, however, were found to be significantly smaller than those calculations using the measured values of $\sigma$ in (16). In later studies [2], [9], however, there were reports that the model provides a better fit to radiometric data over the angular range between 
$10^{\circ}$ and $60^{\circ}$ if the $\cos ^{2} \theta$ factor in the exponential in (15) is suppressed (i.e., using $\exp (-h)$ at all angles). Equation (15) may be rewritten as

with $n=2$ or $n=0$.

$$
R_{r p}(\theta)=R_{p}(\theta) \exp \left(-h \cos ^{n} \theta\right)
$$

\section{II.4. Soil Moisture Retrieval Algorithm}

For each radiometric measurement taken at angle $\theta$, the volumetric soil moisture $m_{v}$ is retrieved by minimizing the difference $D_{1}$ between model calculations and measurements of the soil microwave emissivity e. $D_{1}$ is computed as

$$
D_{1}=\left|e_{\text {mod }}(\theta)-e_{\text {meas }}(\theta)\right|,
$$

If the studied field is homogeneous, the volumetric soil moisture $m_{v}$ may be retrieved by minimizing the quadratic difference $D_{2}$ between model calculations and measurements of the soil microwave emissivity e. $D_{2}$ is computed as

$$
D_{2}=\sum_{i}\left\{e_{\bmod }\left(\theta_{i}\right)-e_{\text {meas }}\left(\theta_{i}\right)\right\}^{2},
$$

where $\Sigma$ is the sum of emissivity data over all available incidence angles $\theta$.

\section{EXPERIMENTAL}

\section{III.1. Instrumentation}

The microwave radiometers, namely the L-band noise-injection radiometer (LNIR) (center frequency $1.41 \mathrm{GHz}$ ) of STI, VAST were used for measuring the microwave emission of soil.

The LNIR is a microprocessor-controlled Dicke-type noise-injection radiometer with center frequency $1.41 \mathrm{GHz}$, sensitivity $\leq 0.3 \mathrm{~K}$ (one second integration time) and short-term instability $\leq 0.3 \mathrm{~K}$. The LNIR has two measurement modes - LOCAL and REMOTE. When working in LOCAL mode, LNIR calculates the average value of the output frequency and the variance for any measuring interval between 1 second and 1024 seconds. When working in REMOTE mode, LNIR should be connected to a personal computer via the RS 232 output port for data recording and post-processing.

\section{III.2. Test Sites and Field Measurements}

The first field experiment was carried out at Hoai Duc Agro-meteorology Center on October 2012. A rough bare soil field about $3 \times 3 \mathrm{~m}^{2}$ was prepared by digging the soil over with hoes (mattocks) and then leveling the experimental plot. The radiometers were calibrated by measuring the emission of the sky and a high-quality microwave absorber with known physical temperature. After calibrating the radiometers, the angular dependence of the microwave brightness temperature of the soil field was measured at $1.4 \mathrm{GHz}$ at horizontal polarization, respectively changing the incidence angle of the antennas. Next, the incidence angle of the LNIR antenna was fixed and several radiometric measurements were performed at different azimuth angles of the antenna in order to check the homogeneity of the experimental plot (soil moisture distribution). Soil samples for estimating the gravimetric soil moisture (GSM) $m_{g}$ at depths $0-2 \mathrm{~cm}, 0-5 \mathrm{~cm}, 5-10 \mathrm{~cm}, 10$ $15 \mathrm{~cm}$ were taken before and after the irrigation. The air temperature and the soil temperature $T_{S}$ at $2 \mathrm{~cm}$ below the surface were also measured. 


\section{RESULTS AND DISCUSSION}

\section{IV.1. Experimental Data}

Immediately after the experiments, the radiometric data for L -band were processed (in MS Excel), and the corresponding microwave brightness temperature and emissivity values were calculated for both experimental sites. To account for the differences in soil physical temperatures, the brightness temperature observations were converted to soil emissivity by dividing by the measured soil temperature $\mathrm{T}_{S}$ instead of the effective soil temperature $T_{\text {eff }}$ (3). To account for the antenna beam width ef-

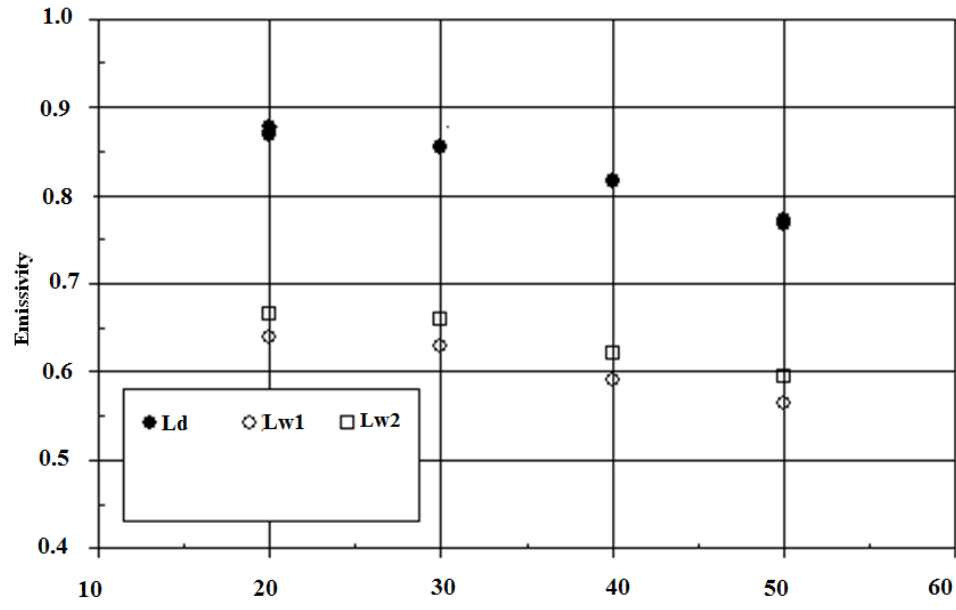

Fig. 1. The angular dependence of the microwave emissivity of the soil fields by L-band fects, only the LNIR data for incidence angles from $20^{\circ}$ to $50^{\circ}$ will be used for soil moisture retrieval. Graphs for illustrating and analyzing the angular dependence of the microwave emissivity of the soil fields were made and shown in Fig. 1 for Hoai Duc site.

The measured gravimetric soil moisture (GSM) $m_{g}$ data were processed and presented in Table 1 (Std is for "standard deviation" and $C V=\left(S t d^{*} 100 /\right.$ Average $)$ is the coefficient of variation). They are used for calculating the volumetric soil moisture $m_{v}[\mathrm{~g} / \mathrm{cm} 3], m_{v}=m_{g}{ }^{*} \rho$, where $\rho$ is the bulk density of the soil.

The soil texture and soil bulk density data given in Table 3, and the GSM data are provided by Geography Institute.

\section{IV.2. Soil Moisture Retrieval}

The program, which applies Wang and Schmugge dielectric model of soil (7)-(14) [6], and the Fresnel coefficients for smooth soil surface (5)-(6), runs under MS Excel and is used for calculating the microwave emission of a bare soil., A surface roughness effects correction is applied (15)-(17). Data about the soil texture (sand and clay content), the bulk density and the temperature of the soil are regarded as input data to the Excel program. The water dielectric constant is calculated according to the model given in Appendix E of [5].

Immediately after the experiments, the experimentally determined soil emissivity values are used for calculating the corresponding surface soil moisture values $V S M_{\text {est }}$ for both sites minimizing the difference $D_{1}(18)$ between model calculations and measurements of the soil microwave emissivity. The results are also presented in Table 2 marked as "L-band estimates, Min $D_{1}, n=2$, $h=0.3$ " for Hoai Duc site, after the soil was compressed. The values of the roughness parameter 
Table 1. Gravimetric soil moisture data for Hoai Duc site

\begin{tabular}{lllllll}
\hline \hline Hoai Duc site & \multicolumn{7}{l}{} \\
\hline \hline D state & Sample1 & Sample2 & Sample3 & & & \\
\hline Depth, cm & GSM [\%] & GSM [\%] & GSM [\%] & Average & Std & CV[\%] \\
$0-2 \mathrm{~cm}$ & 10.98 & 10.43 & 5.01 & 8.80 & 3.30 & 37.5 \\
$0-5 \mathrm{~cm}$ & 19.10 & 21.48 & 21.70 & 20.76 & 1.44 & 6.9 \\
$5-10 \mathrm{~cm}$ & 23.02 & 25.77 & 22.33 & 23.71 & 1.82 & 7.7 \\
$10-15 \mathrm{~cm}$ & 24.98 & 27.21 & 27.53 & 26.57 & 1.39 & 5.2 \\
\hline \hline W2 state & Sample1 & Sample2 & Sample3 & & & \\
\hline Depth, cm & GSM [\%] & GSM [\%] & GSM [\%] & Average & Std & CV[\%] \\
$0-2 \mathrm{~cm}$ & 30.57 & 32.24 & 28.17 & 28.44 & 2.05 & 7.2 \\
$0-5 \mathrm{~cm}$ & 23.48 & 24.99 & 23.12 & 23.45 & 0.30 & 1.3 \\
$5-10 \mathrm{~cm}$ & 24.35 & 25.46 & 25.53 & 20.38 & 0.91 & 4.5 \\
$10-15 \mathrm{~cm}$ & 25.85 & 25.86 & 25.74 & 18.09 & 1.82 & 10.0 \\
\hline \hline
\end{tabular}

Table 2. Measured and estimated volumetric soil moisture data for Hoai Duc site

\begin{tabular}{|l|l|l|l|l|}
\hline Hoai Duc site: VSM data & \multicolumn{1}{|c|}{ D state } & \multicolumn{1}{|c|}{ W1 state } & \multicolumn{1}{|c|}{ W2 state } \\
\hline \hline \multirow{2}{*}{ Ground-truth data } & Layer $0-2 \mathrm{~cm}$ & $0.107(0.088)$ & n.a. & 0.303 \\
\cline { 2 - 5 } & Layer $0-5 \mathrm{~cm}$ & 0.208 & n.a. & 0.239 \\
\hline \hline \multirow{2}{*}{$\begin{array}{l}\text { L-band estimates } \\
\text { Min } D_{1} ; n=2 ; h=0.3\end{array}$} & $20^{\circ}$ & 0.125 & 0.430 & 0.375 \\
\cline { 2 - 5 } & $30^{\circ}$ & 0.115 & 0.375 & 0.320 \\
\cline { 2 - 5 } & $40^{\circ}$ & 0.115 & 0.345 & 0.300 \\
\hline
\end{tabular}

Table 3. Absolute and relative VSM error data for Hoai Duc site

\begin{tabular}{|l|l|l|l|l|}
\hline $\begin{array}{l}\text { Hoai Duc site: } \\
\text { Absolute and relative (\%) }\end{array}$ & VSM errors & D state & W1 state & W2 state \\
\hline \hline \multirow{4}{*}{$\begin{array}{l}\text { L-band estimates } \\
\text { Min D1; } \mathbf{n = 2 ;} \mathbf{h = 0 . 3}\end{array}$} & $20^{\circ}$ & 0.018 & n.a. & 0.072 \\
& & $(16.8 \%)$ & & $(23.8 \%)$ \\
\cline { 2 - 5 } & $30^{\circ}$ & 0.008 & n.a. & 0.017 \\
& & $(7.5 \%)$ & & $(5.6 \%)$ \\
\cline { 2 - 5 } & $40^{\circ}$ & 0.008 & n.a. & -0.003 \\
& & $(7.5 \%)$ & & $(-1 \%)$ \\
\hline
\end{tabular}

$h$ were chosen to be 0.3 and 0.1 , respectively, according to $[7,13,15,17]$, expert knowledge and evaluation of the roughness state of the experimental plots. $n=2$ means that equation (15) is used for surface roughness correction.

The estimated surface soil moisture values $V S M_{e s t}$ are compared with the ground-truth data. The absolute error $\Delta V S M=\left(V S M_{\text {est }}-\operatorname{VSM}(0-2 \mathrm{~cm})\right)$ and the relative error $\delta V S M=$ $\Delta V S M \times 100 / \operatorname{VSM}(0-2 \mathrm{~cm})[\%]$ are calculated and analyzed. They are also given in Table 3 marked as "L-band estimates, Min $D_{1}, n=2, h=0.3$ " for Hoai Duc site after the soil was compressed. 
One of the problems of any remote sensing verification study is the comparison between the remote sensing estimates and the so called ground-truth measurements, often used as "standards". It is well known that the soil moisture has high temporal and spatial variability at almost any scale [11]. For the soil moisture studies, the problem is how to compare the remote sensing estimates with ground-truth measurements (most frequently gravimetric sampling) which are inherently noisy [11]. The traditional instruments used for soil moisture sampling are not quite suitable for ground-truth measurements, especially when the soil moisture profile is very inhomogeneous (i.e., there are large soil moisture gradients with depth), because small error in the depth position of the instrument may produce large soil moisture errors (differences).

\section{CONCLUSIONS}

The experimental results confirmed that the model of Choudhury better fit to radiometric data over the angular range between $20^{\circ}$ and $50^{\circ}$ for $n=0$. The experimental results also confirmed that the effect of roughness is much better fit to radiometric data over the angular range between $20^{\circ}$ and $50^{\circ}$ for $\sigma_{e f f}<\sigma$, where $\sigma$ is the measured standard deviation of surface height. The measured angular dependence of L-band brightness temperature for Hoai Duc site and the estimated $\sigma_{\text {eff }}=0.72 \mathrm{~cm}$ are typical for medium rough agricultural field.

The calculation of L-band volumetric soil moisture compares well with the results from ground-truth measurements for the soil layer $(0-2 \mathrm{~cm})$ with the exception of $D C$ state (dry compressed soil).

Based on the results of the field experiments conducted in Vietnam in 2012 over two experimental sites with different soils, namely sandy loam at Hoai Duc Agrometeorology Center, it may be concluded that the testing of both the radiometric equipment and the method for soil moisture retrieval was very successful, and the main goal of the experiments was fulfilled.

\section{REFERENCES}

[1] A. M. Shutko, Microwave Radiometric Remote Sensing of Waters and Lands, Science Publ., Moscow (in Russian) (1986).

[2] B. J. Choudhury et al., J. Geophys. Res., vol. 84, No. C9, 5699-5706 (1979).-

[3] C. A. Laymon et al., IEEE Trans. Geosci. Remote Sensing, 39(9) (2001) 1844-1858.

[4] E. G. Njoku and J. A. Kong, J. Geophys. Res. 82(20) (1977) 3108-3118.

[5] F. T. Ulaby, R. K. Moore, and A. K. Fung, Microwave Remote Sensing: Active and Passive, vol. III, From Theory to Applications, Artech House, Inc, Dedham, MA (1986).

[6] J. R. Wang and T. J. Schmugge, IEEE Trans. Geosci. Remote Sensing, GE-18(4) (1980) 288-295.

[7] J. R. Wang et al., IEEE Trans. Geosci. Remote Sensing, GE-21(1) (1983) 44-51 .

[8] J. Sabburg, J. A. R. Ball, and N. H. Hancock, IEEE Trans. Geosci. Remote Sensing 35(3) (1997) 784-787 .

[9] J-P. Wigneron, L. Laguerre, and Y. H. Kerr, IEEE Trans. Geosci. Remote Sensing 39(8) (2001) 1697-1707.

[10] K. G. Kostov and B. I. Vichev, Proc. IGARSS'95, July 10-14, 1995, Florence, Italy, 3 (1995) 1991- 1993.

[11] K. R. Bell et al., Water Res. Research 16(4) (1980) 796-810.

[12] M. C. Dobson et al., IEEE Trans. Geosci. Remote Sensing GE-23(1) (1985) 35-46.

[13] National Foundation for Scientific Research Contract No. SS321/1993, "Microwave Methods for Soil and Vegetation Investigations Using Remote Sensing and Contact Equipment”, Final report, October 1996, Sofia, Bulgaria (in Bulgarian) (1996).

[14] T. J. Jackson and P. E. O’Neill, IEEE Trans. Geosci. Remote Sensing GE-24(6) (1986) 920-929.

[15] T. J. Jackson and T. J. Schmugge, IEEE Trans. Geosci. Remote Sensing 27(2) (1989) 225- 235.

[16] T. J. Schmugge and B. J. Choudhury, Radio Science 16(5) (1981) 927-938 .

[17] T. J. Schmugge, P. E. O’Neill, and J. R. Wang, IEEE Trans. Geosci. Remote Sensing GE-24(1) (1986) 12-22. 